\title{
Using Human Approach Paths to Improve Social Navigation
}

\author{
Eleanor Avrunin \\ Carnegie Mellon University \\ Pittsburgh, PA 15213 \\ Email: eavrunin@cs.cmu.edu
}

\author{
Reid Simmons \\ Carnegie Mellon University \\ Pittsburgh, PA 15213 \\ Email: reids@cs.cmu.edu
}

\begin{abstract}
Index Terms - human-robot interaction, navigation, social navigation, proxemics
\end{abstract}

\section{INTRODUCTION}

Many of today's robots are required to navigate autonomously inside buildings in order to carry out their tasks, and a great deal of work focuses on the difficulties of safely and effectively avoiding obstacles while reaching their goals. This is made more challenging by the fact that many of those obstacles are people. Service robot tasks, in particular, may require the robot to navigate toward a human and initiate an interaction, for instance when delivering a package.

Navigating around humans has special complications in addition to simple obstacle avoidance. For instance, there is no need to worry about how a support pillar perceives the robot's path around it, but a person who thinks the robot is coming too close might react badly. The social implications of navigation paths and the expectations they produce are challenges that people handle regularly when moving around each other in their everyday lives. These expectations of others' behaviors are based on non-verbal (or occasionally verbal) communication and knowledge of social rules and patterns. For a robot to be able to follow these rules and engage in this communication would make its behavior appear more predictable and thus more comfortable to the people around it.

We intend to leverage people's customary use of these rules and patterns to help robots follow them. We collect data on human navigation around other humans, from which we create a model that can be used by a robot's path planner to create paths that appear more socially acceptable. We believe that a relatively small sample of human behavioral data can create an effective such model.

While the social aspects of navigation could affect many areas, we have chosen to focus on approaching humans, as in the delivery task mentioned above. This task is part of the larger challenge of starting an interaction with a person who may or may not be expecting the robot's presence [1].

\section{Human PATHS}

We hypothesize that a key factor in determining a person's approach path to another human would be the direction of the other person's attention. Experience suggests that people take very different paths when approaching someone from behind versus a head-on approach. We break this factor down further into body orientation (relative to the person approaching) and head orientation (relative to the body). This gives us nine conditions for our controlled study: the person being approached could stand with the front of the torso, the right side, or the back toward the person approaching, and have their head facing forward, to the left, or to the right in each of the body poses (we omitted poses with the left side facing the approach in order to avoid participant fatigue; we believe they would be analogous to the right side approaches).

\section{A. Data Collection}

We collected data from 7 participants recruited on Carnegie Mellon campus. There were 5 men and 2 women, ranging in age from 23 to 31 . Each participant was instructed to approach a standing researcher from a distance of 4.5 meters (outside the social zone defined in proxemics research [2]) and hand over an empty water bottle without talking or making physical contact with the researcher. The researcher wore large noisecanceling headphones to simulate a distracted and unaware target for the approach. This process was repeated for each of the nine poses, with the order of the poses randomized across participants. The study was recorded using a Kinect sensor and the participants' paths were extracted semi-automatically from the depth data.

\section{B. Results and Observations}

Figure 1 (left and center) shows paths for two conditions, the first with the target person's torso and head facing toward the participant's initial position and the second with the torso and head facing directly away. Recording failed during the second condition for one participant, so only 6 paths are shown there.

As expected, we see distinct differences between the two conditions. In the first condition, the paths are tightly clustered: the participant is in the target's view from the start of the approach and does not deviate significantly from the shortest straight-line path. There is more variation among participants in the second condition, and they do not follow the shortest path. Rather, the participants tend to take curving paths that bring their approach into the target's field of view without coming very close to the target's back. We also note that some participants moved to the target's left, while others went to 

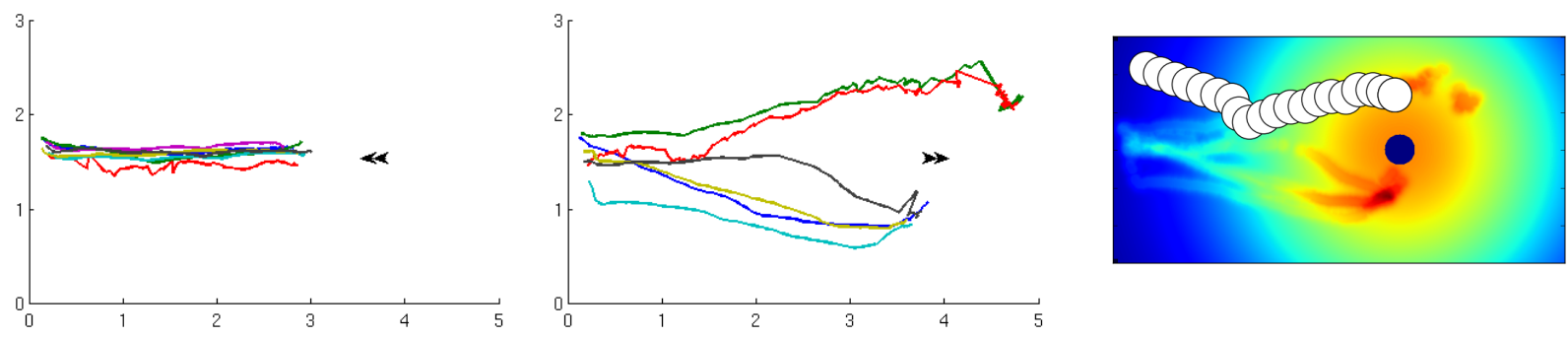

Fig. 1: Human path data for a target with torso and head facing toward the starting position of the approach (left), and with both facing directly away (center). The longer arrow indicates torso orientation, the shorter arrow head. A possible reward function based on the paths and an example social robot path following that reward function (right). Each circle indicates the robot's position after a time step.

the right. In the conditions where the target's back was to the starting position, but the head was turned to one side, the paths go more consistently to that side.

Our results suggest a distinct preference for approach paths that are in the target's field of view for as long as possible. In addition to the social preference for not startling a person one is approaching, participants may expect a target that is aware of the approach to take steps to make it easier, such as turning toward the approaching person. This expectation would mean that visible paths reduce the joint effort of the two agents involved in the approach, making them preferable. This would be analogous to the minimization of joint conversational effort in the linguistic theory of Common Ground [3].

Dautenhahn, et al. [4] compared approaches by a robot from three different starting points and found that participants preferred an approach from the side to a head-on one. In our study, all approaches start from the same point, meaning that an oblique approach in the condition where the target is facing the starting point would require a substantial deviation from the obvious straight-line path.

\section{APPLICATION}

Using these human paths and their goal (the location of the target), we can create a reward function for a robot to follow. One such reward function, using the paths from the second condition discussed above, is shown in Figure 1 (right). The goal is a Gaussian attractor with a hollow center indicating the minimum safe distance from a person. Each human path is smoothed and then a series of smaller Gaussians are placed along its length. The effects of these Gaussians add across the paths, but not within a single path, so that multiple paths indicate a particularly good area, but a path does not reinforce itself.

This reward function, or a similar one, can then be used in several ways to plan a robot's approach path. Figure 1 (right) shows one of the simplest: a greedy path following the reward function from a starting point on the map to the goal. We notice how this path curves and differs from the shortest-path approach the robot otherwise might have taken to reach the person, which would have ended almost directly behind the person. The new path brings the end of the approach into the person's field of view, without passing too close to the person's body.

We are currently preparing an experiment to evaluate the effects of these different paths on people's perceptions of the robot. Participants will be given a task to perform that requires a robot to repeatedly bring them items. For some participants, the robot will simply take the shortest safe path to reach them, while others will be approached using the social reward function in planning the path. We will also investigate the effects of incorporating the social reward when the robot must also avoid non-human obstacles during the approach.

\section{CONCLUSION}

Here we have presented a small study collecting human social navigation data, and a method for adding an approximation of human social rules for approaching others to a robot's autonomous navigation by using a model of those data. For a robot to adhere to those social rules when it moves through human environments would make its navigation more predictable and familiar to humans, who have years of experience with other human agents following those rules, thus potentially improving their acceptance of the robot's presence.

\section{ACKNOWLEDGMENT}

This research was supported by the Department of Defense through the National Defense Science \& Engineering Graduate Fellowship Program.

\section{REFERENCES}

[1] S. Satake, T. Kanda, D. F. Glas, M. Imai, H. Ishiguro, and N. Hagita, "How to approach humans?: Strategies for social robots to initiate interaction," in Proc. 4th ACM/IEEE Intl. Conf. on Human Robot Interaction, ser. HRI '09. New York, NY, USA: ACM, 2009, pp. $109-116$.

[2] E. T. Hall, The Hidden Dimension. Anchor Books, 1966.

[3] H. H. Clark and S. A. Brennan, "Grounding in communication," in Perspectives on socially shared cognition., L. B. Resnick, J. M. Levine, and S. D. Teasley, Eds. APA Books, 1991.

[4] K. Dautenhahn, M. Walters, S. Woods, K. L. Koay, C. L. Nehaniv, A. Sisbot, R. Alami, and T. Siméon, "How may I serve you?: A robot companion approaching a seated person in a helping context," in Proc. 1st ACM SIGCHI/SIGART Conf. on Human-Robot Interaction, ser. HRI '06. New York, NY, USA: ACM, 2006, pp. 172-179. 